\title{
A Two Control Limits Double Sampling Control Chart by Optimizing Producer and Customer Risks
}

\author{
Dradjad Irianto \& Ani Juliani \\ Manufacturing Systems Research Group FTI, Institute of Technology Bandung \\ Email: dradjad@mail.ti.itb.ac.id
}

\begin{abstract}
Standard Shewhart process control chart has been widely used, but it is not sensitive in detecting small shift. A number of alternatives have been proposed to improve the capability of control chart. The double sampling (DS) control chart is aimed at improving the capability to detect any small shift out-of control condition by observing the second sample without interruption. The capabilities of DS control chart were measured as the expected sample size (as a measure of inspection cost) or the control chart power (as a measure for customer risk). Optimization of these criteria is used to determine the control limits. In this paper, we optimize both producer and customer risks under a certain expected number of sample sizes as the constraint. Comparing the result to the previous procedure that only optimize customer risk, the proposed optimization procedure gives the same first control limit but smaller second control limits with higher value of control chart power.
\end{abstract}

Keywords: control chart; customer risk; double sampling; optimization; producer risk.

\section{$1 \quad$ Introduction}

Regardless of how well designed or maintained, any manufacturing process produces inherent or natural variability as a cumulative effect of unavoidable causes. Control chart is one among recognized statistical process control tools that, in general, is proactive and mainly aimed at monitoring the process [1]. A control chart is designed to accurately identify natural variability in a manufacturing process as a result of unassignable (or chance) causes, or a result of assignable (or special) causes, which is considered as out-of-control process. In this respect, the standard Shewhart $\bar{X}$ control chart has been widely used $[1,2]$. Much of its recognition is approved because the Shewhart $\bar{X}$ control chart is simple and effective. The design of Shewhart control chart is simply determined by producer risk $\alpha$ (the risk to decide that the process is out-ofcontrol when it is under-control). At a level of $\alpha=0.0027$, so that the distances of control limits from process mean are three times of sample standard deviation (see e.g. [2]).

This standard Shewhart control chart is, however, not sensitive in detecting small shift that is common in today precise manufacturing processes. This

Received September $2^{\text {nd }}, 2009$, Revised June $8^{\text {th }}, 2010$, Accepted for publication October $28^{\text {th }}, 2010$. 
weakness causes the customer risk or the risk of passing non-conforming output when deciding that the process is under-control when it is out-of-control. A number of alternatives have been proposed to improve the power or sensitivity of control chart in detecting small shift, such as moving average control chart, cumulative sum control chart and control chart with warning limits [2]. We should note that a quick action to false indication of out-of-control process can be misleading. Because of randomness of noise in process data, the presence of an assignable cause is often not immediately observable. It is a mistake to react quickly to an outcome as if it came from a special cause, when actually it is not [3]. This condition is known as false alarm. Quick detection without increasing false alarm rate is desirable. Accordingly, a control chart with warning limit is explored further to get better control on the manufacturing process. A control chart with this objective is useful for continuous flow manufacturing system for high volume production.

Reynolds, et al. [4] proposed a control chart with warning limit known as a variable sampling interval (VSI) control chart. If an out-of-control warning or signal occurs, next sample will be taken in a shorter sampling interval; otherwise it is reasonable to take a longer sampling interval. They also proposed multiple sampling intervals, but only provided numerical example for two sampling interval. Instead of varying sampling interval, Costa [5] proposed a variable sample size (VSS) control chart in dealing with an out-of-control warning or signal. Costa [5] also mentioned a simple model using only two sample sizes. VSI and VSS have the same idea of using a confirmation in the occurrence of out-of-control signal, and both are better (higher control chart power) in detecting out-of-control condition with small shift than standard Shewhart control chart.

The double sampling procedure (DS) uses the both ideas of VSI and VSS simultaneously. In case an out-of-control warning or signal occurs, in addition to the first sample, the second sample is added (similar to the idea of VSS). This second sample with larger sample size is observed with zero or the shortest time interval (similar to the idea of VSI). The DS control chart was firstly proposed by Croasdale [6]. In this first DS control chart, information from the first and second samples is evaluated separately, which thus confirmation is done only with the second sample. Daudin, et al. [7] and Daudin [8] improved Croasdale's DS control chart, and proposed DS control chart that utilizes the information from both samples at the second stage. This larger sample size improved the precision of control chart since it uses smaller sample standard deviation. In estimating the control chart limits, Daudin's DS control chart optimized of the expected sample size. Instead of minimizing the expected sample size, Irianto and Shinozaki [9] maximized the power of control chart to determine the 
control chart limits. He, et al. [10], and Costa and Claro [11] have made further development of DS control chart.

In the economic design of control charts, there are three categories of costs to consider [2], i.e. costs for sampling and sample inspection, costs for investigating out-of-control signal and correcting the deviation, and costs of producing non-conforming products. Accordingly, there are three motivations in designing a control chart and in estimating of control chart parameter, i.e. (i) minimizing the expected number of sampling and inspection, (ii) maximizing capability or probability to detect out-of-control signal, and (iii) minimizing false alarm (out-of control alarm when process is under-control). The second motivation is known as improving the power of control chart so that minimizing customer risk, while the third motivation is for minimizing producer risk. The third motivation is minimizing the time of inoperative system since the process is considered as out-of-control in the presence of common cause (undercontrol). In this paper, we proposed a method to estimate the control chart limits by optimizing the risks of producer and customer. By considering both risks, we can optimize the determination of control chart limits under both conditions, i.e. false alarm condition when process is under-control and delivering nonconforming output under out-of-control process, simultaneously.

After introduction chapter, the outline of this paper includes a brief review on the development of DS control chart, how to estimate the control chart limits by optimizing the power of the test, and how to estimate the control limits by optimizing both producer and costumer risks. At the end we can conclude this paper.

\section{The DS Control Chart Procedures}

Croasdale's DS control chart procedure [6] is described as follows (its scheme is also exhibited in Figure 1):

1. Take concurrently the first sample of size $n_{1}\left(X_{1 i}, i=1,2, \cdots, n_{1}\right)$ and the second sample of size $n_{2}\left(X_{2 i}, i=1,2, \cdots n_{2}\right)$ from a population with mean value $\mu_{0}$ and a known standard deviation $\sigma$.

2. Calculate the first sample mean $\bar{X}_{1}=\sum_{i=1}^{n_{1}} X_{1 i} / n_{1}$.

3. Let $\left[-M_{1}, M_{1}\right]$ are limits for the first stage. If $\frac{\bar{X}_{1}-\mu_{0}}{\sigma / \sqrt{n_{1}}}$ is in $\left[-M_{1}, M_{1}\right]$, the process is considered to be under-control, otherwise calculate the sample 
mean $\bar{X}_{2}=\sum_{i=1}^{n_{2}} X_{2 i} / n_{2}$ and observe the second stage.

4. Let $\left[-M_{2}, M_{2}\right]$ are limits for the second stage. If $\frac{\bar{X}_{2}-\mu_{0}}{\sigma / \sqrt{n_{2}}}<-M_{2}$, or if $\frac{\bar{X}_{2}-\mu_{0}}{\sigma / \sqrt{n_{2}}}>M_{2}$, then the process is considered to be out-of-control, otherwise the process is considered to be under-control.

For a shift from the mean value $\delta=\left(\mu_{0}-\mu\right) / \sigma$, assume the characteristic of output of process follows a normal distribution function $N\left(\mu, \sigma^{2}\right)$, the probability that the process is monitored as under-control is given as follows:

$$
\begin{aligned}
& P=\Phi\left[M_{1}+\delta \sqrt{n_{1}}\right]-\Phi\left[-M_{1}+\delta \sqrt{n_{1}}\right] \\
& +\left\{1-\Phi\left[M_{1}+\delta \sqrt{n_{1}}\right]\right\} \Phi\left[M_{2}+\delta \sqrt{n_{2}}\right]+\Phi\left[-M_{1}+\delta \sqrt{n_{1}}\right] \cdot\left\{1-\Phi\left[-M_{2}+\delta \sqrt{n_{2}}\right]\right\}
\end{aligned}
$$

where $\Phi(\cdot)$ is the cumulative distribution function of standard normal distribution. The average run length is ARL $=1 /(1-P)$, and the expected total sample size is $n_{1}+n_{2} \cdot\left(1-P_{1}\right)$, where $P_{1}=1-\Phi\left[M_{1}+\delta \sqrt{n_{1}}\right]+\Phi\left[-M_{1}+\delta \sqrt{n_{1}}\right]$.

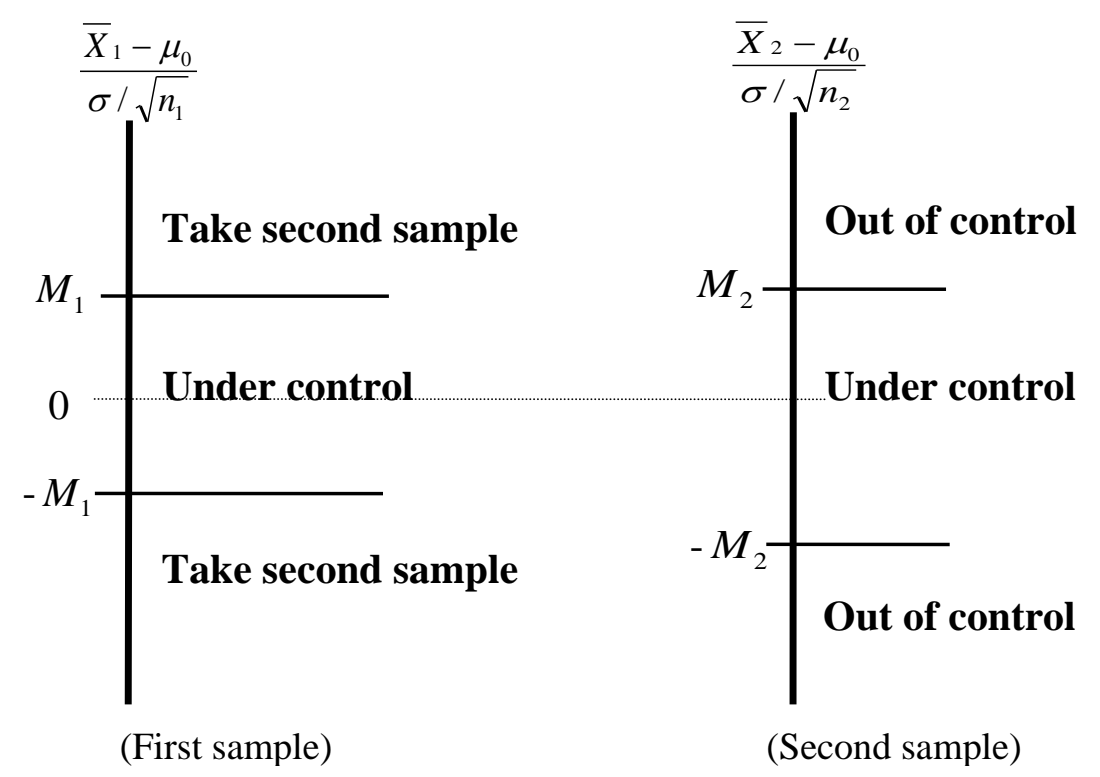

Figure 1 Croasdale's DS control chart procedure. 
Different with Croasdale's procedure, in Daudin's DS control chart [8], the second sample will be observed only if the first sample is signaling a warning, a grey area between under-control and out-of-control decisions as in Figure 2. The procedure is described as follows:

1. Take concurrently the first sample of size $n_{1}, X_{1 i}, i=1,2, \cdots . n_{1}$ and the second sample of size $n_{2}, X_{2 i}, i=1,2, \cdots . n_{2}$ from a population with mean value $\mu_{0}$ and a known standard deviation $\sigma$.

2. Calculate the sample mean $\bar{X}_{1}=\sum_{i=1}^{n_{1}} X_{1 i} / n_{1}$.

3. If $\left(\bar{X}_{1}-\mu_{0}\right) /\left(\sigma / \sqrt{n_{1}}\right)$ is in $I_{1}$ (see Figure 2 ), the process is considered to be under-control.

4. If $\left(\bar{X}_{1}-\mu_{0}\right) /\left(\sigma / \sqrt{n_{1}}\right)$ is in $I_{3}$ (see Figure 2), the process is considered to be out-of-control.

5. If $\left(\bar{X}_{1}-\mu_{0}\right) /\left(\sigma / \sqrt{n_{1}}\right)$ is in $I_{2}$ (see Figure 2 ), calculate the second sample mean $\bar{X}_{2}=\sum_{i=1}^{n_{2}} X_{2 i} / n_{2}$ and observe the second stage.

6. Calculate the total sample mean $\bar{X}=\left(n_{1} \bar{X}_{1}+n_{2} \bar{X}_{2}\right) /\left(n_{1}+n_{2}\right)$ and sample standard deviation $\sigma / \sqrt{n_{1}+n_{2}}$.

7. If $-L<\frac{\bar{X}_{1}-\mu_{0}}{\sigma / \sqrt{n_{1}}}<-L_{1}$ or $L_{1}<\frac{\bar{X}_{1}-\mu_{0}}{\sigma / \sqrt{n_{1}}}<L$, and if $\frac{\bar{X}-\mu_{0}}{\sigma / \sqrt{n_{1}+n_{2}}}<-L_{2}$ or $\frac{\bar{X}-\mu_{0}}{\sigma / \sqrt{n_{1}+n_{2}}}>L_{2}$, then the process is considered to be out-of-control, otherwise the process is considered under-control.

Let $\bar{Z}_{1}=\left(\bar{X}_{1}-\mu_{0}\right) /\left(\sigma / \sqrt{n_{1}}\right)$ and $\bar{Z}=\left(\bar{X}-\mu_{0}\right) /\left(\sigma / \sqrt{n_{1}+n_{2}}\right)$, then the probabilities that the process is considered to be under control by the first sample and after observing the second sample can be formulated as $P_{a 1}=\operatorname{Pr}\left[\bar{Z}_{1} \in I_{1}\right]$ and $P_{a 2}=\operatorname{Pr}\left[\bar{Z}_{1} \in I_{2}\right.$ and $\left.\bar{Z} \in I_{4}\right]$ respectively, and the probability that process under control is $P_{a}=P_{a 1}+P_{a 2}$. 


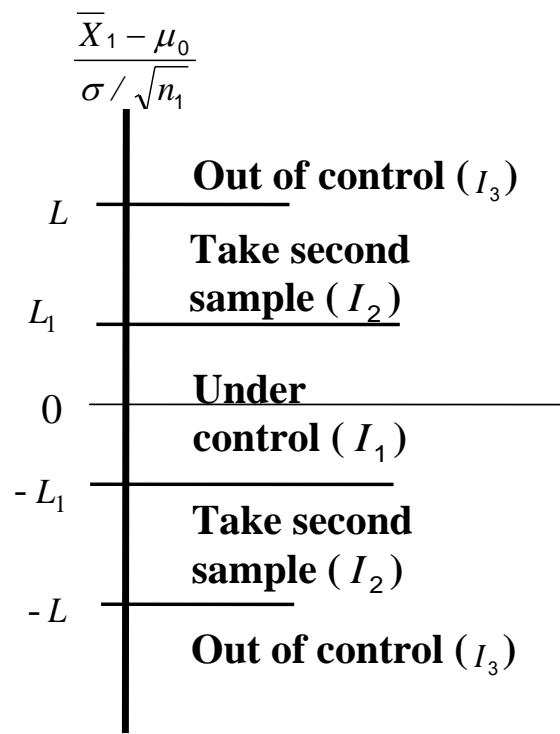

(First sample)

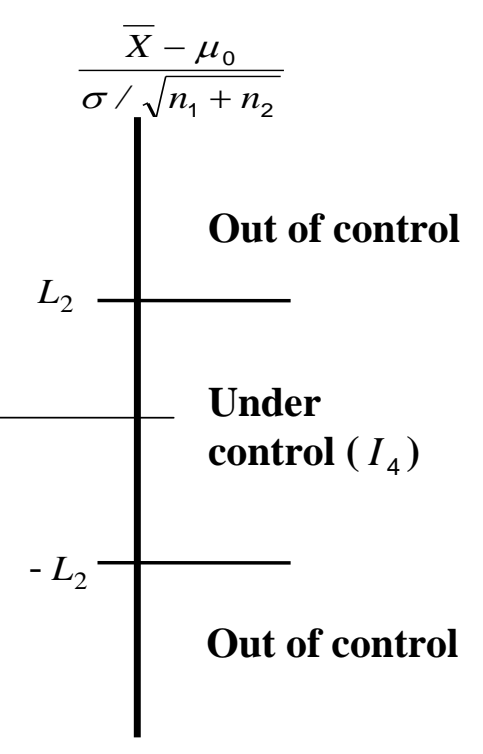

(Second sample)

Figure 2 The Daudin's DS control chart procedure.

For a shift from the mean value $\delta=\left(\mu_{0}-\mu\right) / \sigma$, the probability that the process is considered to be under-control becomes:

$$
\begin{aligned}
& P_{a}=\Phi\left[L_{1}+\delta \sqrt{n_{1}}\right]-\Phi\left[-L_{1}+\delta \sqrt{n_{1}}\right] \\
& +\int_{z \in I_{2}^{*}}\left\{\Phi\left[c L_{2}+r c \delta-z \sqrt{n_{1} / n_{2}}\right]-\Phi\left[-c L_{2}+r c \delta-z \sqrt{n_{1} / n_{2}}\right]\right\} \phi(z) d z
\end{aligned}
$$

where $\phi(\cdot)$ and $\Phi(\cdot)$ are the density and cumulative distribution functions of standard normal distribution respectively, $I_{2}^{*}=\left[-L+\delta \sqrt{n_{1}},-L_{1}+\delta \sqrt{n_{1}}\right) \bigcup\left(L_{1}+\delta \sqrt{n_{1}}, L+\delta \sqrt{n_{1}}\right], r=\sqrt{n_{1}+n_{2}}$, and $c=r / \sqrt{n_{2}}$. The average run length is ARL $=1 /\left(1-P_{a}\right)$, and the expected total sample size is $n_{1}+n_{2} \cdot \operatorname{Pr}\left[\bar{Z}_{1} \in I_{2}\right]$. Irianto and Shinozaki [9] analyzed both DS control charts, and proved the advantage of Daudin's DS control chart compared to Croasdale's DS control chart in detecting out-of-control signals. 


\section{$3 \quad$ Estimating the Limits of DS Control Chart}

There are five parameters required to specify the Daudin's DS control charts, i.e. $L_{1}, L_{2}, L, n_{1}$ and $n_{2}$. Daudin, et al. [7] suggested an optimization procedure for minimizing the expected sample number to be inspected, which thus the motivation is to reduce the inspection cost for monitoring the manufacturing process (producer risk). To find the solution, they proposed a heuristic algorithm as follows:

(i) Determine $n_{1}$ and $n_{2}$.

(ii) For a given value of $L$, both constraints are used to determine the values of $L_{1}$ and $L_{2}$.

(iii) Find the optimal composition of $L_{1}, L_{2}$ and $L$ numerically that minimize the objective function for all possible pairs of $\left(n_{1}, n_{2}\right)$.

Differently, Irianto and Shinozaki [9] considered the power or capability of control chart in detecting the process mean shift. Therefore, the motivation is to minimize risk of not knowing that the process mean has deviated (customer risk) while setting sample sizes $n_{1}$ and $n_{2}$ so that the expected total sample size is fixed (e.g. $n=4$ or 5 as suggested by Shewhart [2]). The optimization is formulated as follows:

$$
\begin{aligned}
& \operatorname{Max}_{\mathrm{L}, \mathrm{L}_{1}, \mathrm{~L}_{2}} 1-\left\{\Phi\left[L_{1}+\delta \sqrt{n_{1}}\right]-\Phi\left[-L_{1}+\delta \sqrt{n_{1}}\right]\right\} \\
& -\int_{z \in I_{2}^{*}}\left\{\Phi\left[c L_{2}+r c \delta-z \sqrt{n_{1} / n_{2}}\right]-\Phi\left[-c L_{2}+r c \delta-z \sqrt{n_{1} / n_{2}}\right]\right\} \phi(z) d z
\end{aligned}
$$

Subject to:

(i) E[total sample size $\left.\mid \mu=\mu_{0}\right]=n$, that is

$$
n_{1}+n_{2} \cdot \operatorname{Pr}\left[\bar{Z}_{1} \in I_{2} \mid \mu=\mu_{0}\right]=n \Leftrightarrow L=\Phi^{-1}\left[\frac{n-n_{1}}{2 n_{2}}+\Phi\left[L_{1}\right]\right] .
$$

(ii) $\operatorname{Pr}\left[\right.$ Out of Control $\left.\mid \mu=\mu_{0}\right]=\alpha$, that is

$$
\begin{aligned}
& 1-\left\{\Phi\left[L_{1}\right]-\Phi\left[-L_{1}\right]\right\} \\
& -\int_{z \in I_{2}^{*}}\left\{\Phi\left[c L_{2}-\sqrt{n_{1} / n_{2}} z\right]-\Phi\left[-c L_{2}-\sqrt{n_{1} / n_{2}} z\right]\right\} \phi(z) d z=\alpha
\end{aligned}
$$

From the first constraint, $L$ can be expressed in terms of $L_{1}$, which then it reduces the number of decision variables. Since the left hand side of the second 
constraint is an increasing function of $L_{2}$, then $L_{2}$ can be uniquely determined for fixed $L_{1}$ and $L$.

Usually, standard Shewhart chart is used as the basis for comparison. The standard Shewhart $\bar{X}$ control chart use $n=5$ and $L=3$, which thus the producer risk $\alpha$ is set at 0.0027 . For a shift $\delta=\left|\mu_{1}-\mu_{0}\right|=0.5$ and 1.0 , the power of the test are 0.0064 and 0.0228 , respectively. Table 1 shows some control limits of DS control charts for some pairs of $n_{1}$ and $n_{2}$ but still give an expected sampling number $n=5$. It is clear that the DS control chart gives better power than the standard Shewhart $\bar{X}$ control chart. Accordingly, the out-of-control signal will occur in a shorter interval than the standard Shewhart $\bar{X}$ control chart. However, it should be noted that the expected sample size increases as the shift of process mean gets larger.

Table 1 Power of DS control chart for some pairs of sample sizes.

\begin{tabular}{cccccc}
\hline \multirow{2}{*}{ Sample size } & \multirow{2}{*}{$L_{1}$} & \multirow{2}{*}{$L$} & $L_{2}$ & \multicolumn{2}{c}{ Power } \\
\cline { 5 - 6 }$n_{1}=4 ; n_{2}=2 ;$ & 0.673 & 3.3057 & 3.0720 & 0.0357 & 0.2766 \\
$n=5$ & 0.674 & 3.6057 & 3.0149 & 0.0375 & 0.2882 \\
& 0.6744 & $\infty$ & 2.9999 & 0.0379 & 0.2910 \\
\hline$n_{1}=4 ; n_{2}=3 ;$ & 0.966 & 3.3854 & 3.0557 & 0.0440 & 0.3459 \\
$n=5$ & 0.967 & 3.7058 & 3.0087 & 0.0461 & 0.3577 \\
& 0.9674 & $\infty$ & 2.9961 & 0.0467 & 0.3606 \\
\hline$n_{1}=4 ; n_{2}=5 ;$ & 1.280 & 3.4575 & 3.0135 & 0.0611 & 0.4662 \\
$n=5$ & 1.281 & 3.7271 & 2.9754 & 0.0637 & 0.4762 \\
& 1.2815 & $\infty$ & 2.9593 & 0.0647 & 0.4801 \\
\hline$n_{1}=4 ; n_{2}=6 ;$ & 1.381 & 3.4261 & 2.9966 & 0.0683 & 0.5069 \\
$n=5$ & 1.382 & 3.6110 & 2.9590 & 0.0711 & 0.5158 \\
& 1.3829 & $\infty$ & 2.9292 & 0.0733 & 0.5225 \\
\hline
\end{tabular}

Table 1 also shows that maximizing power leads to higher value of $L_{1}$ (with lower value of $L_{2}$ ). Using the function in the first constraint, the higher value of $L_{1}$ implies to higher value of $L$, which is limited to $L=\infty$. If $L$ is very large, then it is no longer necessary as an out-of-control limit at the first stage. Irianto [12] proposed a revised the DS control chart by eliminating $L$, and its scheme is shown in Figure 3. 


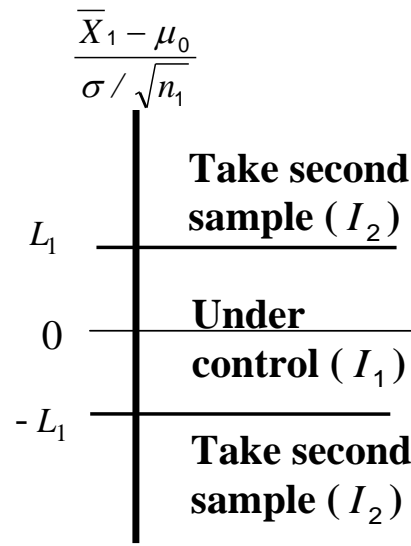

(First sample)

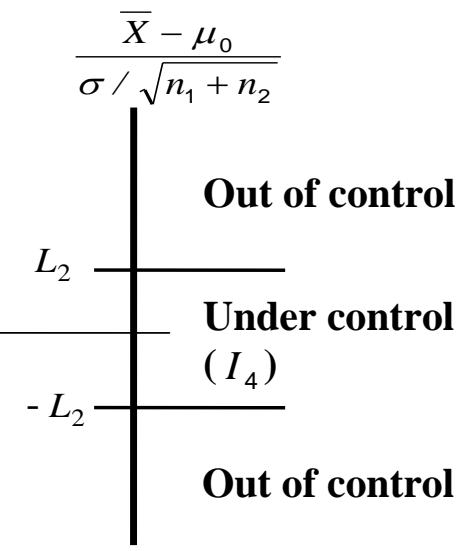

(Second sample)

Figure 3 Revised DS control chart.

Table $2 P_{a}$ of revised DS control chart for some pairs of sample sizes.

\begin{tabular}{ccccc}
\hline \multirow{2}{*}{ Sample size } & $L_{1}$ & $L_{2}$ & \multicolumn{2}{c}{ Power $\delta=\left|\mu_{1}-\mu_{0}\right|$} \\
\cline { 4 - 5 } & & & $\delta=\mathbf{0 . 5}$ & $\delta=\mathbf{1 . 0}$ \\
\hline$n_{1}=4 ; n_{2}=2 ;$ & 0.672 & 2.7832 & 0.040270 & 0.336669 \\
$n=5$ & 0.674 & 2.7823 & 0.040281 & 0.336828 \\
& 0.6744 & 2.7821 & 0.040286 & 0.336879 \\
\hline & 0.965 & 2.6491 & 0.049780 & 0.424442 \\
$n_{1}=4 ; n_{2}=3 ;$ & 0.966 & 2.6486 & 0.049783 & 0.424494 \\
$n=5$ & 0.967 & 2.6481 & 0.049785 & 0.424545 \\
& 0.9674 & 2.6478 & 0.049775 & 0.424579 \\
\hline & 1.279 & 2.4718 & 0.066476 & 0.536531 \\
$n_{1}=4 ; n_{2}=5 ;$ & 1.280 & 2.4712 & 0.066471 & 0.536484 \\
$n=5$ & 1.281 & 2.4706 & 0.066461 & 0.536421 \\
& 1.2815 & 2.4703 & 0.066460 & 0.536399 \\
\hline & 1.380 & 2.4063 & 0.073804 & 0.567977 \\
$n_{1}=4 ; n_{2}=6 ;$ & 1.381 & 2.4057 & 0.073788 & 0.567860 \\
$n=5$ & 1.382 & 2.4050 & 0.073777 & 0.567753 \\
& 1.3829 & 2.4043 & 0.073766 & 0.567647 \\
\hline & & & &
\end{tabular}

Accordingly the optimization of power in (3) can be reformulated as follows: 


$$
\begin{aligned}
& \operatorname{Max}_{\mathrm{L}_{1}, \mathrm{~L}_{2}} 1-\Phi\left[\left[L_{1}+\delta \sqrt{n_{1}}\right]+\Phi\left[-L_{1}+\delta \sqrt{n_{1}}\right]\right. \\
& \quad-\left\{1-\Phi\left[L_{1}+\delta \sqrt{n_{1}}\right]+\Phi\left[-L_{1}+\delta \sqrt{n_{1}}\right]\right\} .\left\{\Phi\left[L_{2}+\delta \sqrt{n_{1}+n_{2}}\right]-\Phi\left[-L_{2}+\delta \sqrt{n_{1}+n_{2}}\right]\right\}
\end{aligned}
$$

Subject to:

(i) $\mathrm{E}$ [total sample size $\left.\mid \mu=\mu_{0}\right]=n$, and then

$$
n_{1}+n_{2} \cdot \operatorname{Pr}\left[\bar{Z}_{1} \in I_{2} \mid \mu=\mu_{0}\right]=n \Leftrightarrow n_{1}+n_{2} \cdot\left\{1-\Phi\left[L_{1}\right]+\Phi\left[-L_{1}\right]\right\}=n .
$$

(i) $\operatorname{Pr}\left[\right.$ Out of Control $\left.\mid \mu=\mu_{0}\right]=\alpha$, and then

$$
\left\{1-\Phi\left[L_{1}\right]+\Phi\left[-L_{1}\right]\right\} .\left\{1-\Phi\left[L_{2}\right]+\Phi\left[-L_{2}\right]\right\}=\alpha .
$$

Table 2 shows some control limits of DS control charts for some pairs of $n_{1}$ and $n_{2}$ but still give an expected sampling number $n=5$. Compared to Table 1 , the revised DS control chart provides higher power, which means higher capability in detecting shift of process mean.

\section{$4 \quad$ Considering Producer and Customer Risk}

As discussed in the first chapter, there are three motivations in designing a control chart and in estimating of control chart parameter, i.e. (i) minimizing the expected number of sampling and inspection, (ii) maximizing the power of control chart or minimizing customer risk, and (iii) minimizing false alarm rate or minimizing producer risk. Considering first and third motivations, Standard Shewhart control chart [2] is set by determining a fixed sample size $n$ (suggested 4 or 5) and a false alarm rate $\alpha=0.0027$. Minimizing cost for inspection in the first motivation will lead to minimize the sample size (in single sampling) or to minimize the expected number of sample (in double sampling). This first motivation was used by Daudin [8] in order to estimate control chart limits. Instead of using the first motivation, Irianto and Shinozaki [9] and Irianto [12] second motivation.

In manufacturing cycle, the process usually starts under-control and is monitored using control chart. If an assignable cause occurs, corrective action must be done to eliminate the cause [13]. However, an out-of-control signal can occur without an assignable cause, known as false alarm signal. In this case an inexperience operator may shut the system down and tries to find the assignable cause. Since it is a false alarm, process interruption for nothing costs the producer. Accordingly, the false alarm rate or producer risk should be minimized (the third motivation), and it can be done concurrently with the second motivation (i.e. minimizing customer risk). 
Based on the revised DS control chart the false alarm will occur at the second stage. The false alarm rate $\alpha$ is affected by control limits $L_{1}$ and $L_{2}$. Similarly, the rate of customer risk $\beta$ is also affected by control limits $L_{1}$ and $L_{2}$. Accordingly, we can develop an optimization model as a function of control limits $L_{1}$ and $L_{2}$ as follows

$$
\operatorname{Min} C_{1} \alpha+C_{2} \beta
$$

Subject to:

$$
\begin{aligned}
& \text { E [total sample number } \left.\mid \mu=\mu_{O}\right]=n \\
& n_{1}+n_{2} \cdot \operatorname{Pr}\left[\bar{Z}_{1} \in I_{2} \mid \mu=\mu_{O}\right]=n \Leftrightarrow n_{1}+n_{2} \cdot\left\{1-\Phi\left[L_{1}\right]+\Phi\left[-L_{1}\right]\right\}=n,
\end{aligned}
$$

where $C_{1}$ and $C_{2}$ are costs related to for producer and customer risks respectively.

The expected values of both risks are as follows:

- $\operatorname{Pr}\left[\right.$ Out of control $\left.\mid \mu=\mu_{O}\right]=\alpha$ or

$$
\left\{1-\Phi\left[L_{1}\right]+\Phi\left[-L_{1}\right]\right\} \cdot\left\{1-\Phi\left[L_{2}\right]+\Phi\left[-L_{2}\right]\right\}=\alpha
$$

- $\operatorname{Pr}\left[\right.$ Under- control $\left.\mid \mu=\mu_{1}\right]=\beta$ or

$$
\begin{aligned}
& \Phi\left[L_{1}+\delta \sqrt{n_{1}}\right]-\Phi\left[-L_{1}+\delta \sqrt{n_{1}}\right]+\left\{1-\Phi\left[L_{1}+\delta \sqrt{n_{1}}\right]+\Phi\left[-L_{1}+\delta \sqrt{n_{1}}\right]\right\} . \\
& \left\{\Phi\left[L_{2}+\delta \sqrt{n_{1}+n_{2}}\right]-\Phi\left[-L_{2}+\delta \sqrt{n_{1}+n_{2}}\right]\right\}=\beta .
\end{aligned}
$$

Optimization for equation (5) is not straightforward, and thus a heuristic approach is used as in Figure 4. Table 3 shows the optimization results for $2 C_{1}=C_{2}$ and $C_{1}=2 C_{2}$.

Table 3 Control limits $L_{l}$ and $L_{2}$.

\begin{tabular}{ccccc|ccc}
\hline \multirow{2}{*}{ Sample size } & $\boldsymbol{\Delta}$ & \multicolumn{3}{c|}{$2 C_{1}=C_{2}$} & \multicolumn{3}{c}{$C_{1}=2 C_{2}$} \\
\cline { 3 - 8 } & & $L_{1}$ & $L_{2}$ & Power & $L_{1}$ & $L_{2}$ & Power \\
\hline$n_{1}=4 ; n_{2}=2 ;$ & 0.5 & 0.6745 & 0 & - & 0.6745 & 1.4781 & 0.2722 \\
$n=5$ & 1.0 & 0.6745 & 0.9763 & 0.8474 & 0.6745 & 1.5455 & 0.7445 \\
\hline$n_{1}=4 ; n_{2}=3 ;$ & 0.5 & 0.9674 & 0 & - & 0.9674 & 1.3258 & 0.2703 \\
$n=5$ & 1.0 & 0.9674 & 0.9665 & 0.8111 & 0.9674 & 1.4926 & 0.7448 \\
\hline$n_{1}=4 ; n_{2}=5 ;$ & 0.5 & 1.2816 & 0 & - & 1.2816 & 1.1931 & 0.2499 \\
$n=5$ & 1.0 & 1.2826 & 1.0525 & 0.7449 & 1.2816 & 1.5152 & 0.7117 \\
\hline$n_{1}=4 ; n_{2}=6$ & 0.5 & 1.3830 & 0 & - & 1.383 & 1.1656 & 0.2387 \\
$n=5$ & 1.0 & 1.3830 & 1.1130 & 0.7170 & 1.383 & 1.5517 & 0.6925 \\
\hline
\end{tabular}




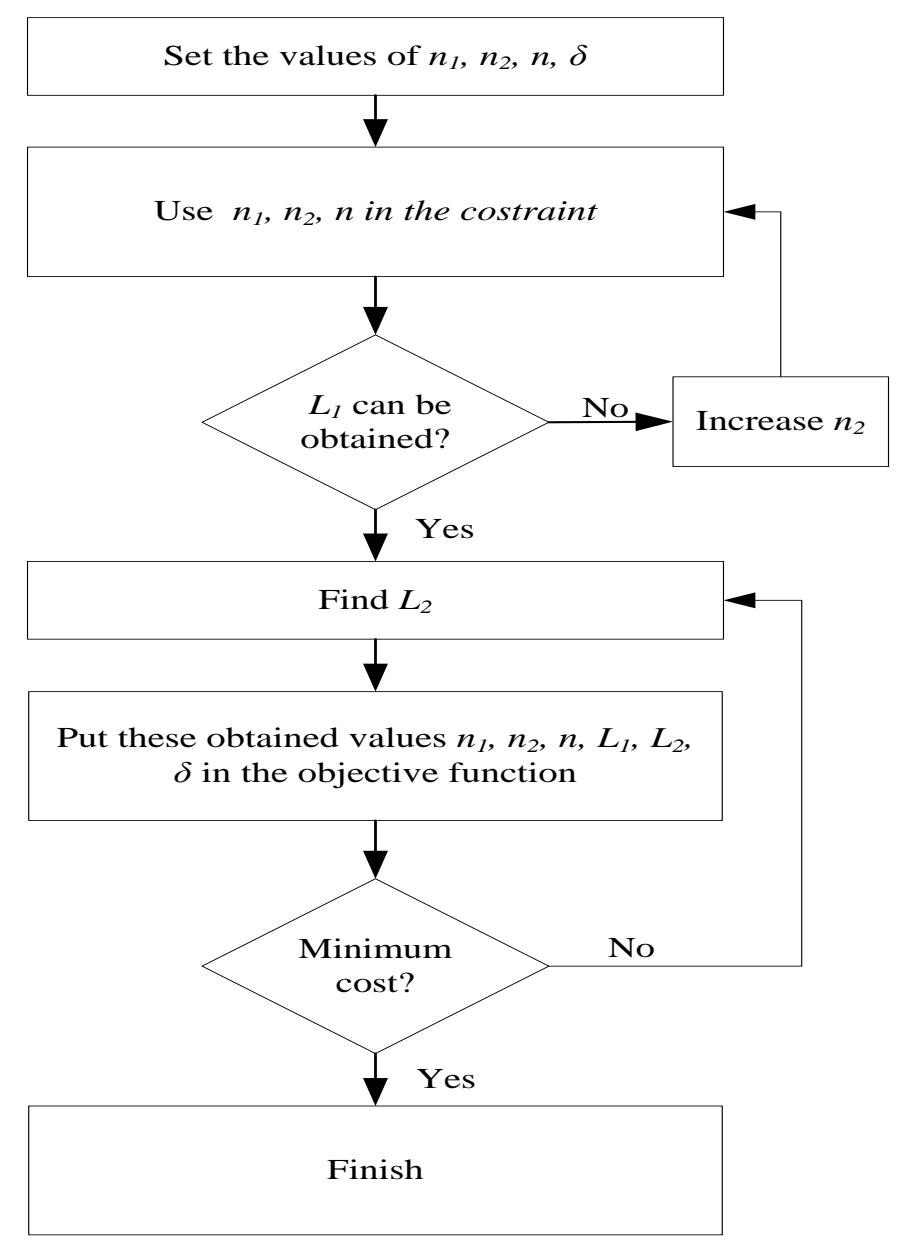

Figure 4 Algorithm for finding the optimum solution.

Higher values of $C_{1}$ means that the manufacturing system is expensive, and thus process interruption should be minimized. On the other hand, higher value of $C_{2}$ means the risk at the customer is high, and thus $\beta$ risk should be minimize. A manufacturer is considered more internal oriented will have $C_{1}>C_{2}$. The reason of this orientation usually is for maximizing the utilization of facilities, and at the end will result in internal efficiency. Otherwise a manufacturer is considered as external oriented if $C_{1}<C_{2}$. This is an orientation towards customer satisfaction. In case where $2 C_{1}=C_{2}$, as in Table 
3 , there is no feasible solution for $\delta<0.5$, while in case of $C_{1}=2 C_{2}$ feasible solution can be obtained.

As mentioned previously, there are three motivations in designing a control chart and in estimating of control chart parameter. This proposed method clearly considered the second and third motivations, i.e. maximizing capability or probability to detect out-of-control signal $(1-\beta)$, and minimizing producer risk $(\alpha)$. Both risks are expressed in control chart limits $L_{1}$ and $L_{2}$. Compared to Table 2 (only considering customer risk), with the same arrangements of sample size $n_{1}, n_{2}$, and $n$, the result (in Table 3 ) gives the same first control limits $\left(L_{1}\right)$ and smaller second control limits $\left(L_{2}\right)$. Since the expected number of inspected items is determined by the value of the first control limits $\left(L_{1}\right)$, the proposed method has the same expected cost for inspection (as the first motivation). The results also give higher control chart power $(1-\beta)$, which thus reduces the customer risk. However, smaller second control limits $\left(L_{2}\right)$ surely increases the false alarm rate. For example if $n_{1}=4, n_{2}=2$, and $n=5$, the optimum value of $L_{1}=0.6745$ and $L_{2}=0.9763$, then the power is $1-\beta=0.8474$ (compared to 0.3369 from Table2) and the producer risk $\alpha=0.1645$ (compared to 0.0027 constraint of Table 2). If cost at producer is higher (e.g. $C_{1}=2 C_{2}$ ) the $L_{2}$ increases to 1.5455 , then the power is $1-\beta$ decreases to 0.7445 and the producer risk $\alpha$ decreases to 0.0611 .

\section{Conclusion}

Despite of the advantage of giving higher capability in detecting out-of-control signal of mean shift, the DS procedure needs a complicated calculation in estimating its control limits. Efficiency of the calculation is improved by changing the optimization problem that implies on the resulted control chart limits and control chart power. This paper developed an optimization procedure for DS control chart under a motivation toward both internal efficiency and external customer satisfaction, simultaneously. This paper is focused on detecting the process mean shift as the main objectives in on-line quality control. Further research will be done that will give focus on variance control.

\section{Acknowledgement}

The authors highly acknowledge comments of reviewers that contribute to a significant improvement of this paper. 


\section{References}

[1] Stuart, M., Mullins, E. \& Drew, E., Statistical Quality Control and Improvement, European Journal of Operational Research, 88, 203-214, 1996.

[2] Montgomery, D.C., Introduction to Statistical Quality Control, $6^{\text {th }}$ Edition, John Wiley \& Sons Pte., Singapore, 2009.

[3] Deming, W.E., The New Economics for Industry, Government, Education, MIT-CAES, Cambridge, USA, 1993.

[4] Reynolds, M.R. Jr., Amin, R.W., Arnold, J.C. \& Nachlas, J.A., $\bar{X}$ Charts with Variable Sampling Interval, Technometrics, 30(2), 181-192, 1988.

[5] Costa, A.F.B., $\bar{X}$ Charts with Variable Sample Size, Journal of Quality Technology, 26(3), 155-163, 1994.

[6] Croasdale, R., Control Charts for a Double-Sampling Scheme Based On Average Production Run Lengths, International Journal of Production Research, 12(5), 585-592, 1974.

[7] Daudin, J.J., Duby, C. \& Trecourt, P., Plans de Controle Double Optimaux (Maitrise des Procedes et Controle de Reception), Rev. Statistique Appliquee, 38(4), 45-59, 1990.

[8] Daudin, J.J., Double Sampling $\bar{X}$ Charts, Journal of Quality Technology, 24(2), 78-87, 1992.

[9] Irianto, D. \& Shinozaki, N., An Optimal Double Sampling $\bar{X}$ Control Chart, International Journal of Industrial Engineering - Theory, Applications and Practice, 5(3), 226-234, 1998.

[10] He, D., Grigoryan, A. \& Sigh, M., Design of Double- and TripleSampling X-bar Control Chart Using Genetic Algorithms, International Journal of Production Research, 40(6), 1387-1404, 2002.

[11] Costa, A.F.B. \& Claro, F.A.E., Double Sampling X Control Chart for a First-Order Autoregressive Moving Average Process Model, International Journal of Advanced Manufacturing Technology, on-line edition, 2007.

[12] Irianto, D., A Revised Double Sampling Control Chart, Proceedings of $14^{\text {th }}$ Asia Pacific Quality Organization International Conference, Teheran, 2008 .

[13] Stoumbos, Z.G. \& Reynolds, M.R. Jr., Economic Statistical Design of Adaptive Control Schemes for Monitoring the Mean and Variance: An Application to Analyzers, Non Linear Analysis: Real World Applications 6, 817-844, 2005. 